\title{
Podocalyxin promotes cisplatin chemoresistance in osteosarcoma cells through phosphatidylinositide 3-kinase signaling
}

\author{
ZHENG HUANG $^{1}$, YANKE HUANG ${ }^{2}$, HONGTAO HE $^{1}$ and JIANGDONG NI ${ }^{1}$ \\ ${ }^{1}$ Department of Orthopaedics, The Second Xiangya Hospital, Central South University, Changsha, Hunan 410011; \\ ${ }^{2}$ Child Health Division, Shenzhen Nanshan District Mother-Child Hospital, Shenzhen, Guangdong 518021, P.R. China
}

Received July 27, 2014; Accepted April 14, 2015

DOI: $10.3892 / \mathrm{mmr} .2015 .3859$

\begin{abstract}
Osteosarcoma (OS) is the most common type of primary bone malignancy. The use of multiagent, intensive chemotherapy has markedly improved the long-term survival rate of patients with OS. However, chemoresistance continues to be the principal reason for poor survival and disease recurrence in patients with OS. Innate or acquired resistance to cisplatin, which is one of the most effective drugs against OS, is common. Understanding the molecular basis underlying cisplatin chemoresistance in OS cells may serve as a basis for the identification of novel therapeutic targets and biomarkers. High expression levels of podocalyxin (PCX) have been shown to be correlated with poor outcome in various types of cancer. A recent study suggested that PCX may contribute to cancer chemoresistance. The present study aimed to explore the role of PCX in OS by determining its effects on cisplatin chemoresistance in OS cells. Stable overexpression and knockdown of PCX were performed in MG-63 and U2OS human OS cell lines. Overexpression of PCX in the two cell lines significantly increased the half maximal inhibitory concentration $\left(\mathrm{IC}_{50}\right)$ of cisplatin, cell colony formation, phosphatidylinositide 3-kinase (PI3K) activity and Akt phosphorylation at serine 473, and decreased cisplatin-induced cell apoptosis. Furthermore, the effects of PCX were largely attenuated by treatment with the selective PI3K inhibitor BKM120. Conversely, knockdown of PCX expression markedly decreased the $\mathrm{IC}_{50}$ of cisplatin, cell colony formation, PI3K activity and Akt phosphorylation at serine 473, and increased cisplatin-induced cell apoptosis. In conclusion, the present study was the first, to the best of our knowledge, to provide evidence that PCX promotes cisplatin chemoresistance in OS cells through a PI3K-dependent mechanism. The results of the present study provided novel insight
\end{abstract}

Correspondence to: Dr Jiangdong $\mathrm{Ni}$, Department of Orthopaedics, The Second Xiangya Hospital, Central South University, 139 Renmin Road, Changsha, Hunan 410011, P.R. China E-mail: nijiangdong@yahoo.com

Key words: osteosarcoma, podocalyxin, chemoresistance, cisplatin, phosphatidylinositide 3-kinase not only into the functional role of PCX in cancer, but also into the molecular mechanisms underlying OS chemoresistance.

\section{Introduction}

Osteosarcoma (OS) is the most common type of primary bone malignancy, and the eighth most common type of cancer in children, comprising $2.4 \%$ of all malignancies in pediatric patients and $\sim 35 \%$ of all bone cancers worldwide (1). The five-year survival rate of patients with OS has significantly improved over recent decades to $60-70 \%$, since the advent of combinatorial chemotherapy (2). However, survival has since leveled, despite advances in therapeutic strategies (3). Standard chemotherapy of OS is based on a combination of several drugs: Neoadjuvant therapy with methotrexate, cisplatin and doxorubicin, followed by surgery and post-operative chemotherapy with methotrexate, cisplatin, doxorubicin, cyclophosphamide and vincristine (4). The use of cisplatin, which is an effective anti-tumor agent with a wide spectrum of activity against human solid tumors (5), has been shown to be a useful chemotherapeutic strategy for pre-operative induction therapy of OS. Patients who responded well to pre-operative cisplatin chemotherapy were also shown to have an improved survival rate (6). Furthermore, the inclusion of cisplatin has been shown to be associated with a better outcome for high-grade OS (7). Intrinsic or acquired chemoresistance is the predominant reason for poor survival and disease relapse in patients with OS (8). Recently, novel molecular-targeted drugs have emerged; however, these drugs have not been well established for the treatment of OS (9). In addition, the molecular mechanisms underlying OS chemoresistance remain to be elucidated. Therefore, identification of factors that contribute to OS chemoresistance and elucidation of the underlying mechanisms is essential for the development of novel therapeutic strategies.

Podocalyxin (PCX) is a highly glycosylated and sialylated transmembrane protein. PCX is a CD34 ortholog that is usually expressed on hematopoietic stem cells, hemangioblasts, vascular endothelial cells, podocytes and a subset of neural progenitors (10). Aberrant PCX expression has been reported in leukemia $(11,12)$, undifferentiated thyroid carcinoma (13) and renal cell carcinoma (14). High protein expression levels of PCX have been shown to be correlated with poor outcome in a subset of breast carcinoma, and have also been associated with increased aggressiveness of breast and prostate cancer cells $(15,16)$. Recently, it has been demonstrated that 
PCX promotes astrocytoma cell survival against temozolomide-induced apoptotic stress (17), thus suggesting that PCX may also contribute to cancer chemoresistance. The present study aimed to explore the role of PCX in OS by determining its effect on cisplatin chemoresistance in OS cells.

\section{Materials and methods}

Cell lines, plasmids and reagents. MG-63 (CRL-1427) and U2OS (HTB-96) human OS cell lines were purchased from the American Tissue Culture Collection (Manassas, VA, USA). The cells were grown in Dulbecco's modified Eagle's medium (InvitrogenLife Technologies, Carlsbad,CA,USA) supplemented with $10 \%$ fetal bovine serum (Invitrogen Life Technologies) and $100 \mathrm{U} / \mathrm{ml}$ penicillin-streptomycin (Sigma-Aldrich, Beijing, China) in an incubator with a humidified atmosphere of $95 \%$ air and $5 \% \mathrm{CO}_{2}$ at $37^{\circ} \mathrm{C}$. Human full-length PCX cDNA (SC302189; Origene Technologies, Inc., Beijing China) was sub-cloned into a pcDNA 3.1 expression vector (Invitrogen Life Technologies) at the KpnI and NotI sites. A human PCX short hairpin (sh)RNA plasmid (RHS3979-98487921; 5'-AGTTCATCCCATTTG TCCT-3') was purchased from Open Biosystems (Huntsville, AL, USA). Mouse anti-human anti-PCX (3D3) monoclonal (cat. no. 39-3800) antibody and Lipofectamine ${ }^{\circledR} 2000$ transfection reagent were purchased from Invitrogen Life Technologies (Carlsbad, CA, USA). Selective phosphatidylinositide 3-kinase (PI3K) inhibitor BKM120 (cat. no. sc-364437A), and rabbit anti-human anti-Akt serine 473 (ser473) (cat. no. sc-1618) and rabbit anti-human anti-phosphorylated (p)-Akt (ser473) (cat. no. sc-101629) polyclonal antibodies were purchased from Santa Cruz Biotechnology, Inc. (Dallas, TX, USA). All primary antibodies were incubated with the membrane at a dilution of 1:500 for $1 \mathrm{~h}$ in Tris-buffered saline with Tween 20 containing $5 \%$ skimmed milk powder for blocking at room temperature. All secondary antibodies were purchased from Jackson ImmunoResearch Laboratories, Inc. (West Grove, PA, USA). The TiterTACS in situ Apoptosis Detection kit (cat. no. 4822-96-K) was purchased from R\&D Systems (Minneapolis, MN, USA). The PI3K Activity ELISA kit (cat. no. K-1000s) was purchased from Echelon Biosciences, Inc. (Salt Lake City, UT, USA). Cisplatin, puromycin, G418 and all chemicals of reagent grade were purchased from Sigma-Aldrich (St. Louis, MO, USA).

Transfection and lentiviral transduction. The PCX expression construct was transfected into the MG-63 and U2OS cells using Lipofectamine ${ }^{\circledR} 2000$ transfection reagent, according to the manufacturer's instructions. Pools of stable transductants were generated via selection with G418 $(700 \mu \mathrm{g} / \mathrm{ml})$, according to the manufacturer's instructions. Lentiviral transduction was performed in the MG-63 and U2OS cells. Lentiviral particles were packaged with vector psPAX2 and vector pMD2.G, according to the manufacturer's instructions (Open Biosystems). A control virus containing a scrambled shRNA sequence, which did not lead to the specific degradation of any cellular mRNA, was used as a negative control for PCX-shRNA lentiviral particles. Pools of stable transductants were generated via selection with puromycin $(5 \mu \mathrm{g} / \mathrm{ml})$.

Western blot analysis. The cells were dissolved in $250 \mu \mathrm{l}$ 2X SDS loading buffer (62.5 mM Tris-HCl, pH 6.8, 2\%
SDS, 25\% glycerol, 5\% 2-mercaptoethanol; Sigma-Aldrich) and incubated at $95^{\circ} \mathrm{C}$ for $10 \mathrm{~min}$ The supernatant collected following centrifugation at $2,000 \mathrm{x}$ g for $15 \mathrm{~min}$ at $4^{\circ} \mathrm{C}$ was used for protein determination using the Coomassie blue method. Equal amounts of protein for each sample were separated by $10 \%$ SDS-PAGE and blotted onto polyvinylidene difluoride microporous membranes (EMD Millipore, Billerica, MA, USA). The membranes were then incubated for $1 \mathrm{~h}$ with the primary antibodies at a 1:500 dilution and then washed. The membranes were subsequently incubated with the horseradish peroxidase-conjugated secondary antibodies $(1: 4,000)$ for $1 \mathrm{~h}$. The blots were visualized using a GE Healthcare Enhanced Chemiluminescence kit (GE Healthcare, Little Chalfont, UK). Three independent experiments were performed for each western blot analysis. Western blots were quantified using ImageJ software version 1.42 (National Institutes of Health, Bethesda, MD, USA).

Cisplatin chemosensitivity/chemoresistance assay. The cells were plated in duplicates in 96-well plates at a density of $5 \times 10^{3}$ cells/well. Following a $24-\mathrm{h}$ incubation, the medium was replaced with fresh medium with or without various concentrations of cisplatin $(0.1,0.25,0.5,1.0,1.5,3.0,6.0$, 15.0, 30.0 and $55.0 \mathrm{mM})$. For BKM120 treatment, cells were treated with BKM120 $(50 \mu \mathrm{M})$ for $48 \mathrm{~h}$. Cell viability was assayed six days later, using a modified MTT assay (Sigma-Aldrich) as previously described (18). The 96-well plate used was purchased from Thermo Fisher Scientific (Beijing, China) and the microplate reader (SpectraMax Plus 384) was purchased from Molecular Devices, LLC (Beijing, China). The half maximal inhibitory concentration $\left(\mathrm{IC}_{50}\right)$ was defined as the concentration resulting in a $50 \%$ reduction in growth, as compared with that of the control cells.

Cell colony formation assay. Colony formation assays were performed as previously described (19). Briefly, the cells were treated with cisplatin $(5 \mathrm{mM})$ at $37^{\circ} \mathrm{C}$ for $48 \mathrm{~h}$, then trypsinized (Sigma-Aldrich), re-seeded in $100-\mathrm{mm}$ plates $\left(1 \times 10^{3}\right.$ cells/plate) and cultured at $37^{\circ} \mathrm{C}$ for 2 weeks. For BKM120 treatment, cells were treated with BKM120 $(50 \mu \mathrm{M})$ for $48 \mathrm{~h}$. The plates were subsequently fixed with cold methanol (Sigma-Aldrich) at $-20^{\circ} \mathrm{C}$ for $30 \mathrm{~min}$, stained with crystal violet $(0.1 \%)$ (Sigma-Aldrich) and finally washed with water in order to remove background staining. Images were captured using a scanner (InGenius3; Syngene Inc., Frederick, MD, USA), and all colonies $>1.5 \mathrm{~mm}$ in diameter were counted.

Cell apoptosis assay. The cells were cultured at $9 \times 10^{4}$ cells/well in 96-well tissue culture plates and treated with cisplatin $(5 \mathrm{mM})$ at $37^{\circ} \mathrm{C}$ for $48 \mathrm{~h}$. For BKM120 treatment, cells were treated with BKM120 $(50 \mu \mathrm{M})$ for $48 \mathrm{~h}$. The rate of cell apoptosis was measured at $48 \mathrm{~h}$ using the microplate reader-based TiterTACS in situ Apoptosis Detection kit, according to the manufacturer's instructions. The SpectraMax Plus 384 microplate reader was used to measure optical density. Each experiment was repeated three times in duplicates.

PI3K activity assay. PI3K activity was determined using the PI3K Activity ELISA kit, according to the manufacturer's 
A

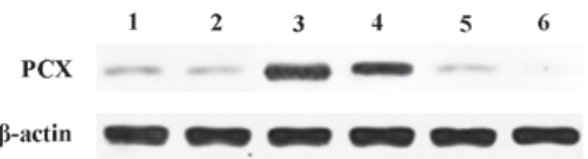

B
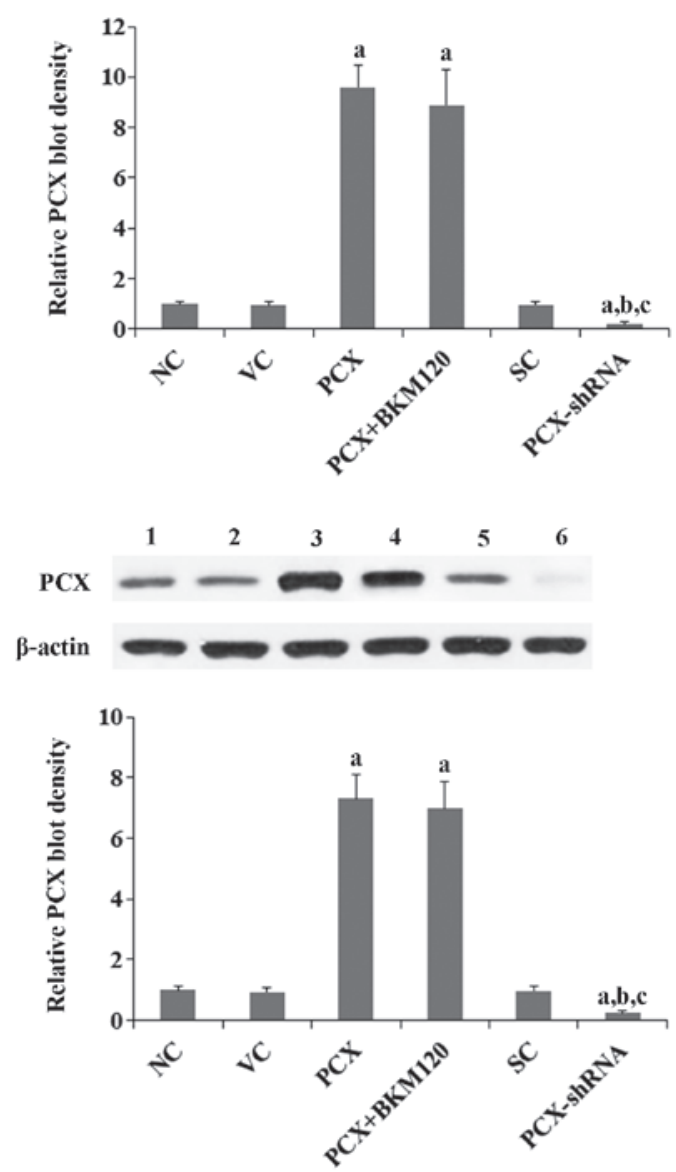

Figure 1. Expression of PCX in OS cells following overexpression or knockdown of PCX. Expression of PCX was detected in (A) MG-63 and (B) U2OS human OS cells by western blot analysis. Lanes: 1, NC; 2, VC; 3 , PCX; 4, PCX + BKM120; 5, SC; and 6, PCX-shRNA. $\beta$-actin was used as a loading control. The density of the PCX blot was normalized against that of the $\beta$-actin blot in order to obtain the relative PCX blot density, which was expressed as a fold change relative to that of the NC group (designated as 1). Three independent experiments were performed for each western blot analysis. Values are expressed as the mean + standard deviation. ${ }^{\mathrm{a}} \mathrm{P}<0.05$, vs. the controls ( $\mathrm{NC}, \mathrm{VC}$ and $\mathrm{SC}$ ); ${ }^{\mathrm{b}} \mathrm{P}<0.05$, vs. the $\mathrm{PCX}$ group; ${ }^{\mathrm{C}} \mathrm{P}<0.05$, vs. the PCX + BKM120 group. Groups: NC, normal control cells; VC, cells stably transfected with empty pcDNA3.1 vector; PCX, cells stably transfected with PCX; PCX + BKM120, cells stably transfected with PCX and treated with the phosphatidylinositide 3-kinase inhibitor BKM120 $(50 \mu \mathrm{M})$ for $48 \mathrm{~h}$; SC, cells stably transduced with scramble control shRNA; and PCX-shRNA, cells stably transduced with PCX-shRNA. PCX, podocalyxin; OS, osteosarcoma; shRNA, small hairpin RNA.

instructions $(20,21)$. For BKM120 treatment, cells were treated with BKM120 $(50 \mu \mathrm{M})$ for $48 \mathrm{~h}$. For direct functional assessment of PI3K activity, PI3K was isolated by immunoprecipitation using an anti-PI3K antibody (cat. no. 06-195; EMD Millipore) to the p85 adapter subunit. The ability of the co-precipitated p110 catalytic subunit to convert standard phosphatidylinositol 4,5-bisphosphate to phosphatidylinositol 3,4,5-trisphosphate (PIP3) in a kinase reaction was assessed by measuring the generated PIP3 by ELISA. The SpectraMax Plus 384 microplate reader was used to measure optical density. Each experiment was repeated three times in duplicates.
A

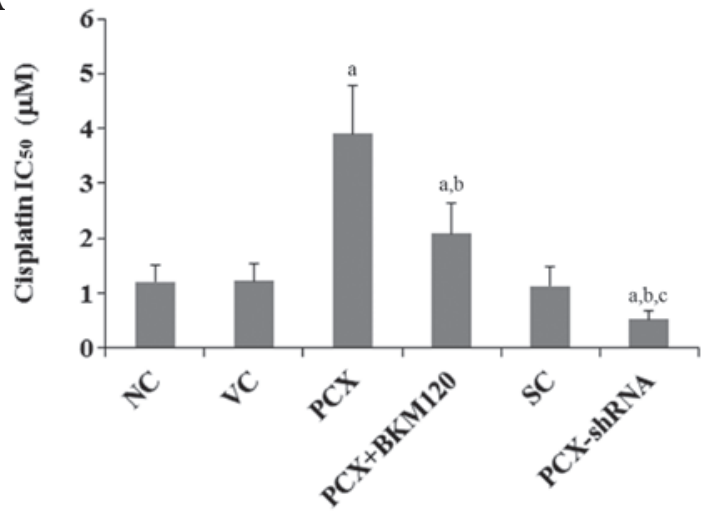

B

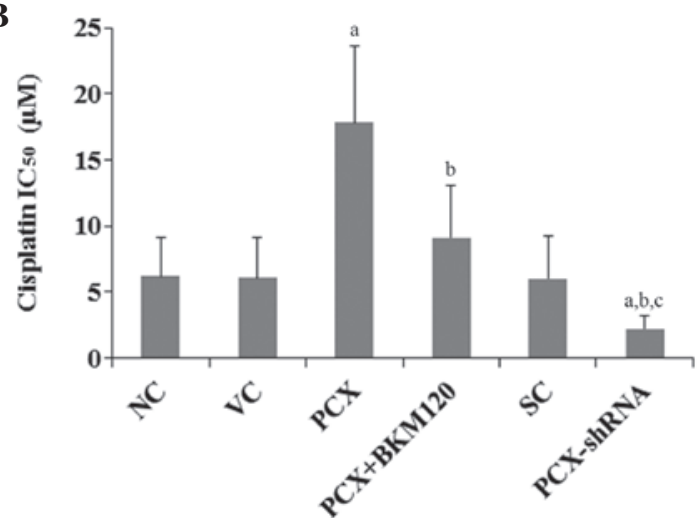

Figure 2. Effects of PCX on the $\mathrm{IC}_{50}$ of cisplatin in (A) MG-63 and (B) U2OS human OS cells. The cells were treated with or without various concentrations of cisplatin for six days, and the $\mathrm{IC}_{50}$ values were determined in the following groups: NC, normal control cells; VC, cells stably transfected with empty pcDNA3.1 vector; PCX, cells stably transfected with PCX; PCX + BKM120, cells stably transfected with PCX and treated with the phosphatidylinositide 3-kinase inhibitor BKM120 $(50 \mu \mathrm{M})$ for $48 \mathrm{~h}$; SC, cells stably transduced with scramble control shRNA; and PCX-shRNA, cells stably transduced with PCX-shRNA. Each experiment was repeated three times in duplicate. Values are expressed as the mean + standard deviation. ${ }^{\mathrm{a}} \mathrm{P}<0.05$, vs. the controls $(\mathrm{NC}$, $\mathrm{VC}$ and $\mathrm{SC}$ ); ${ }^{\mathrm{b}} \mathrm{P}<0.05$, vs. the PCX group; ${ }^{\mathrm{P}} \mathrm{P}<0.05$, vs. the PCX + BKM120 group. PCX, podocalyxin; OS, osteosarcoma; shRNA, small hairpin RNA; $\mathrm{IC}_{50}$, half maximal inhibitory concentration.

Statistical analysis. Statistical analyses were performed using SPSS for Windows 19.0 (International Business Machines, Armonk, NY, USA). Values are expressed as the mean \pm standard deviation. Comparisons of the means among multiple groups were performed by one-way analysis of variance followed by post hoc pairwise comparisons using Tukey's tests. A two-tailed $\mathrm{P}<0.05$ was considered to indicate a statistically significant difference.

\section{Results}

Overexpression and knockdown of PCX in human OS cells. The MG-63 and U2OS human OS cell lines were used as cell models in the present study. As shown in Fig. 1, PCX was constitutively expressed in the two cell lines. PCX was overexpressed or knocked down in the cells by stable transfection with a PCX expression vector or lentiviral transduction of PCX-shRNA, respectively. PCX was overexpressed by 9.6- and 7.3-fold in the MG-63 and U2OS cells transfected with the PCX expression vector, respectively, as compared with that in the control 
A

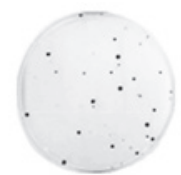

NC

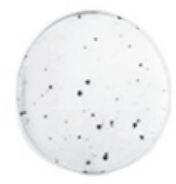

VC

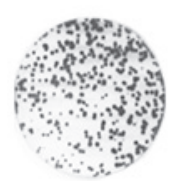

PCX

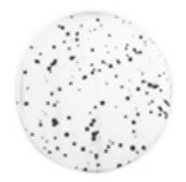

PCX+BKM120

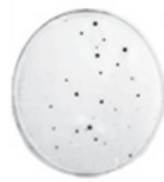

SC

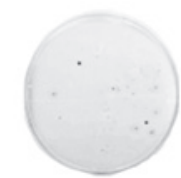

PCX-shRNA

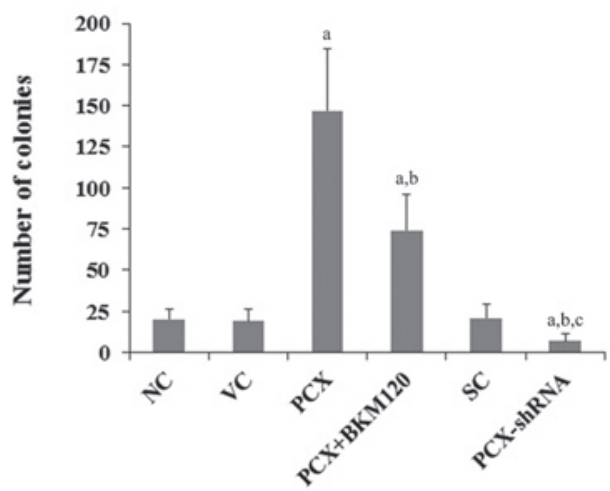

B

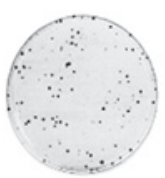

$\mathrm{NC}$

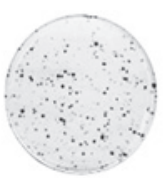

VC

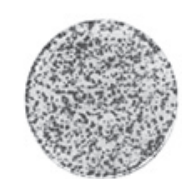

PCX

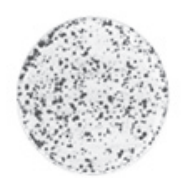

PCX+BKM120

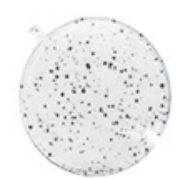

SC

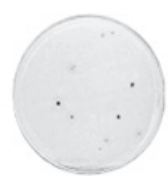

PCX-shRNA

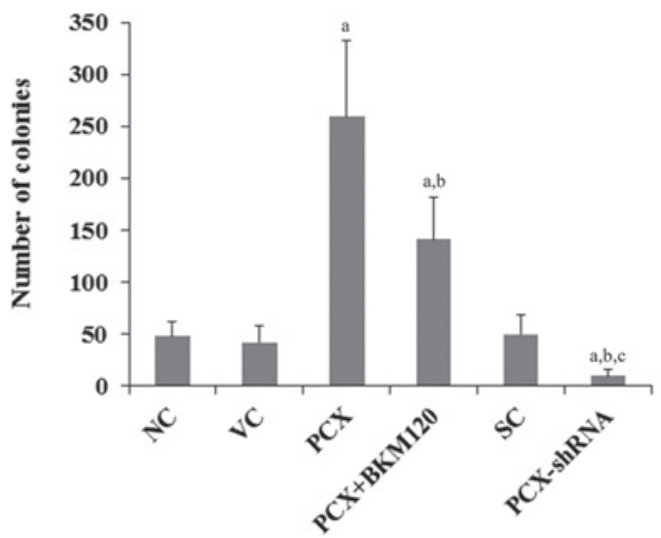

Figure 3. Effects of PCX on cell colony formation in OS cells treated with cisplatin. (A) MG-63 and (B) U2OS human OS cells were treated with $5 \mu$ M cisplatin for $48 \mathrm{~h}$ and then cultured for two weeks. The colonies were stained with crystal violet and counted in the following groups: $\mathrm{NC}$, normal control cells; $\mathrm{VC}$, cells stably transfected with empty pcDNA3.1 vector; PCX, cells stably transfected with PCX; PCX + BKM120, cells stably transfected with PCX and treated with the phosphatidylinositide 3-kinase inhibitor BKM120 (50 $\mu \mathrm{M})$ for $48 \mathrm{~h}$; SC, cells stably transduced with scramble control shRNA; and PCX-shRNA, cells stably transduced with PCX-shRNA. Each experiment was repeated three times in duplicate. Values are expressed as the mean + standard deviation. ${ }^{\mathrm{a}} \mathrm{P}<0.05$, vs. the controls (NC, VC and SC); ${ }^{\mathrm{b}} \mathrm{P}<0.05$, vs. the PCX group; ${ }^{\mathrm{C}} \mathrm{P}<0.05$, vs. the $\mathrm{PCX}+\mathrm{BKM} 120$ group. Magnification, $\mathrm{x} 5$. PCX, podocalyxin; OS, osteosarcoma; shRNA, small hairpin RNA.

cells. Conversely, the endogenous expression levels of PCX were knocked down by $\sim 80$ and $75 \%$ in the MG-63 and U2OS cells transduced with PCX-shRNA, respectively (Fig. 1). A preliminary study using the selective PI3K inhibitor BKM120 had suggested that PCX may regulate OS cell chemoresistance predominantly through a PI3K-dependent mechanism (data not shown); therefore, the present study used the selective PI3K inhibitor BKM120 $(50 \mu \mathrm{M})$ in all experiments. As shown in Fig. 1, treatment with BKM120 had no significant effect on the expression levels of PCX in the two cell lines.

Effects of PCX on cisplatin chemoresistance in OS cells. To explore the effects of PCX on OS chemoresistance, the present study examined the $\mathrm{IC}_{50}$ values of cisplatin on OS cells. As shown in Fig. 2, following six days of cisplatin treatment, the cisplatin $\mathrm{IC}_{50}$ values on MG-63 and U2OS cells were 1.2 and $6.2 \mu \mathrm{M}$, respectively. Overexpression of PCX significantly increased the $\mathrm{IC}_{50}$ values to 3.9 and $17.8 \mu \mathrm{M}$, respectively, which was reduced by $\sim 50 \%$ following treatment with BKM120 $(50 \mu \mathrm{M})$ (Fig. 2). Conversely, knockdown of PCX expression markedly decreased the $\mathrm{IC}_{50}$ values of cisplatin on MG-63 and U2OS cells to 0.5 and $2.1 \mu \mathrm{M}$, respectively (Fig. 2).

Effects of PCX on OS cell colony formation following cisplatin treatment. To determine the effects of PCX on the long-term survival of OS cells, colony formation assays were performed in MG-63 and U2OS cells treated with $5 \mathrm{mM}$ cisplatin for $48 \mathrm{~h}$. After two weeks, the cells were fixed and stained to visualize 

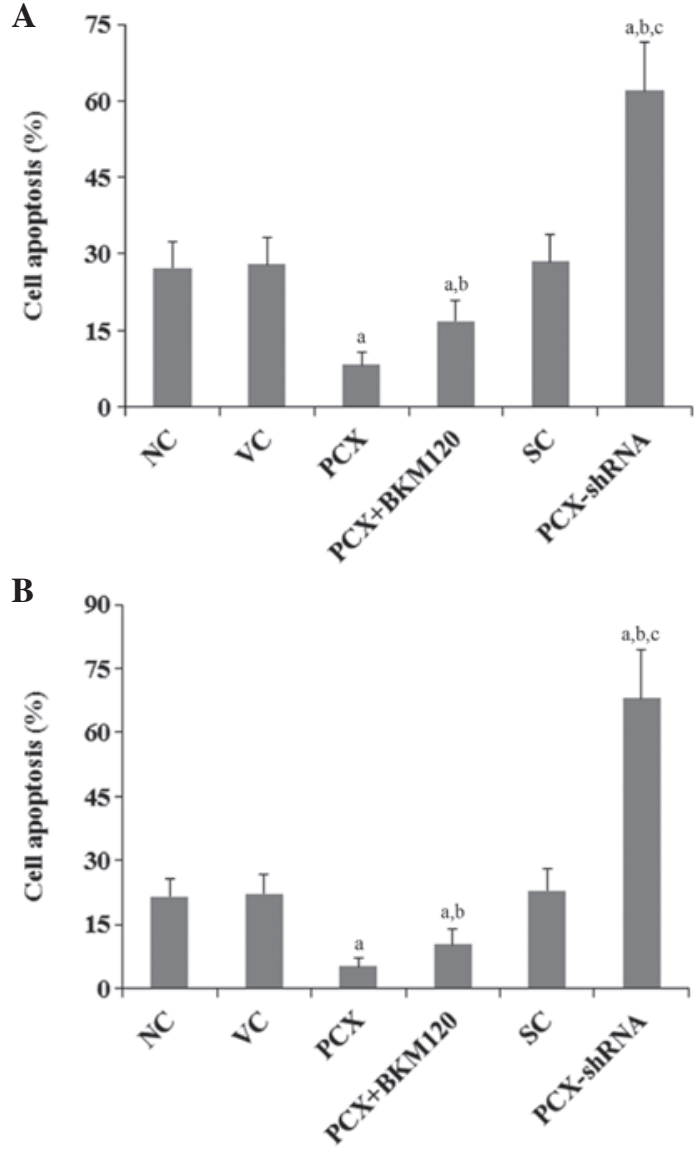

Figure 4. Effects of PCX on cell apoptosis in OS cells treated with cisplatin. (A) MG-63 and (B) U2OS human OS cells were treated with $5 \mu \mathrm{M}$ cisplatin for $48 \mathrm{~h}$. Cell apoptosis was measured using a microplate reader-based TiterTACS in situ Apoptosis Detection kit in the following groups: NC, normal control cells; VC, cells stably transfected with empty pcDNA3.1 vector; PCX, cells stably transfected with PCX; PCX + BKM120, cells stably transfected with PCX and treated with the phosphatidylinositide 3-kinase inhibitor BKM120 $(50 \mu \mathrm{M})$ for $48 \mathrm{~h}$; SC, cells stably transduced with scramble control shRNA; and PCX-shRNA, cells stably transduced with PCX-shRNA. Each experiment was repeated three times in duplicate. Values are expressed as the mean + standard deviation. ${ }^{\mathrm{a}} \mathrm{P}<0.05$, vs. the controls ( $\mathrm{NC}, \mathrm{VC}$ and $\mathrm{SC}$ ); ${ }^{\mathrm{b}} \mathrm{P}<0.05$, vs. the PCX group; ${ }^{\mathrm{P}}<0.05$, vs. the PCX + BKM120 group; PCX, podocalyxin; OS, osteosarcoma; shRNA, small hairpin RNA.

the colonies. As shown in Fig. 3, the number of cell colonies in the MG-63 and U2OS cells was 20 and 48, respectively. Overexpression of PCX significantly increased the number of colonies to 147 and 259 , respectively $(\mathrm{P}<0.05)$, which was reduced by $\sim 50 \%$ following treatment with BKM120 $(50 \mu \mathrm{M})$ (Fig. 3). Conversely, knockdown of PCX expression markedly decreased the number of colonies in the MG-63 and U2OS cells to 7 and 10, respectively (Fig. 3).

Effects of PCX on cisplatin-induced OS cell apoptosis. The present study also examined the effects of PCX on cisplatin-induced apoptosis in OS cells. Under normal culture conditions, overexpression and knockdown of PCX had no significant effects on OS cell apoptosis $(\mathrm{P}>0.05)$, as compared with that of the control cells (data not shown). Following $48 \mathrm{~h}$ of cisplatin treatment $(5 \mathrm{mM})$, the percentages of apoptotic MG-63 and U2OS cells were 27.3 and $21.5 \%$, respectively (Fig. 4). Overexpression of PCX markedly decreased the percentage of apoptotic cells to 8.3 and $5.1 \%$, respectively,
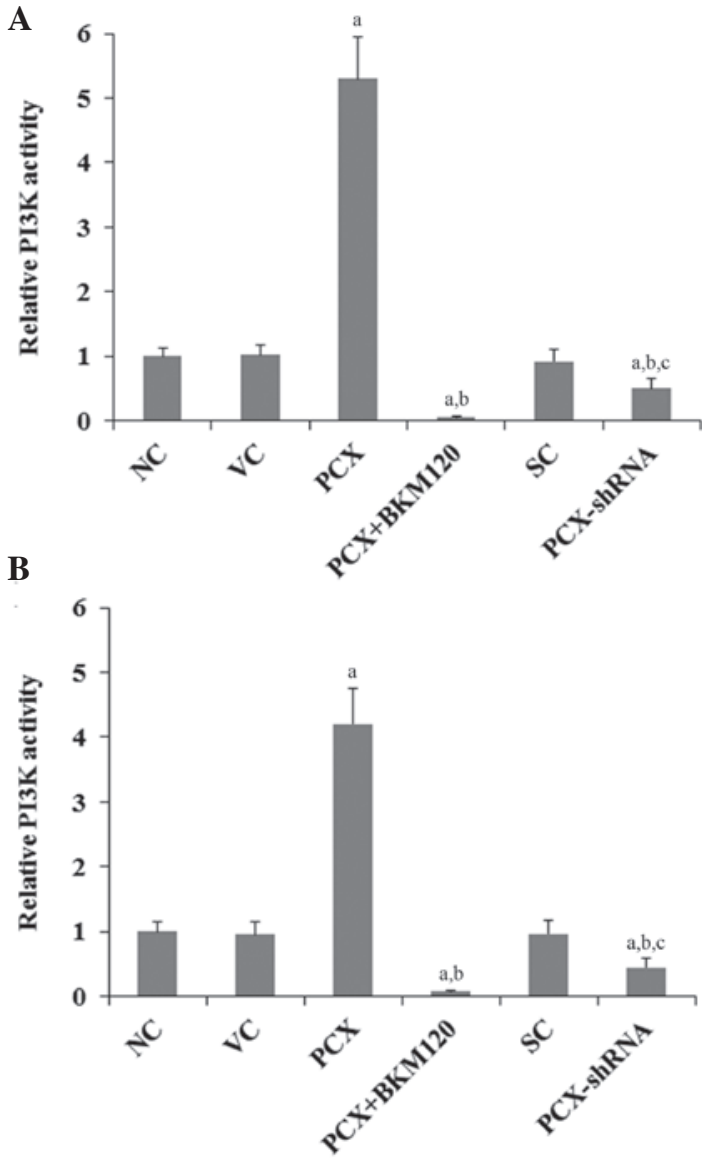

Figure 5. Effects of PCX on PI3K activity in OS cells. In (A) MG-63 and (B) U2OS human OS cells, PI3K activities were determined using a PI3K Activity ELISA kit in the following groups: NC, normal control cells; VC, cells stably transfected with empty pcDNA3.1 vector; PCX, cells stably transfected with PCX; PCX + BKM120, cells stably transfected with PCX and treated with the PI3K inhibitor BKM120 (50 $\mu \mathrm{M})$ for $48 \mathrm{~h}$; SC, cells stably transduced with scramble control shRNA; and PCX-shRNA, cells stably transduced with PCX-shRNA. Each experiment was repeated three times in duplicate. Values are expressed as the mean + standard deviation. ${ }^{a} \mathrm{P}<0.05$, vs the controls ( $\mathrm{NC}, \mathrm{VC}$ and $\mathrm{SC}$ ); ${ }^{\mathrm{b}} \mathrm{P}<0.05$, vs. the $\mathrm{PCX}$ group; ${ }^{\mathrm{C}}<0.05$, vs. the PCX+BKM120 group. PCX, podocalyxin; OS, osteosarcoma; shRNA, small hairpin RNA; PI3K, phosphatidylinositide 3-kinase.

which was partially reversed by treatment with BKM120 $(50 \mu \mathrm{M})$ to 16.9 and $10.2 \%$, respectively (Fig. 4). Conversely, knockdown of PCX markedly increased the percentage of apoptotic cells to 62.0 and $67.9 \%$, respectively (Fig. 4 ; $\mathrm{P}<0.05$ ).

Effects of PCX on PI3K activity and phosphorylation of Akt in OS cells. The results of the present study suggested that PCX promotes cisplatin chemoresistance in OS cells, predominantly through a PI3K-dependent mechanism. Previous studies have demonstrated that the PI3K/Akt pathway is important for cancer cell chemoresistance (22-25). Therefore, the present study examined the effects of PCX on the activity of PI3K and phosphorylation of Akt in OS cells. As shown in Fig. 5, overexpression of PCX increased PI3K activity by 5.3-fold in MG-63 cells and by 4.2-fold in U2OS cells, as compared with that in the control cells, which was attenuated by BKM120 treatment $(50 \mu \mathrm{M})$. Conversely, knockdown of PCX expression decreased PI3K activity by $~ 50 \%$ in the two cell lines (Fig. 5; P<0.05). A similar trend was observed for the phosphorylation at serine 473 

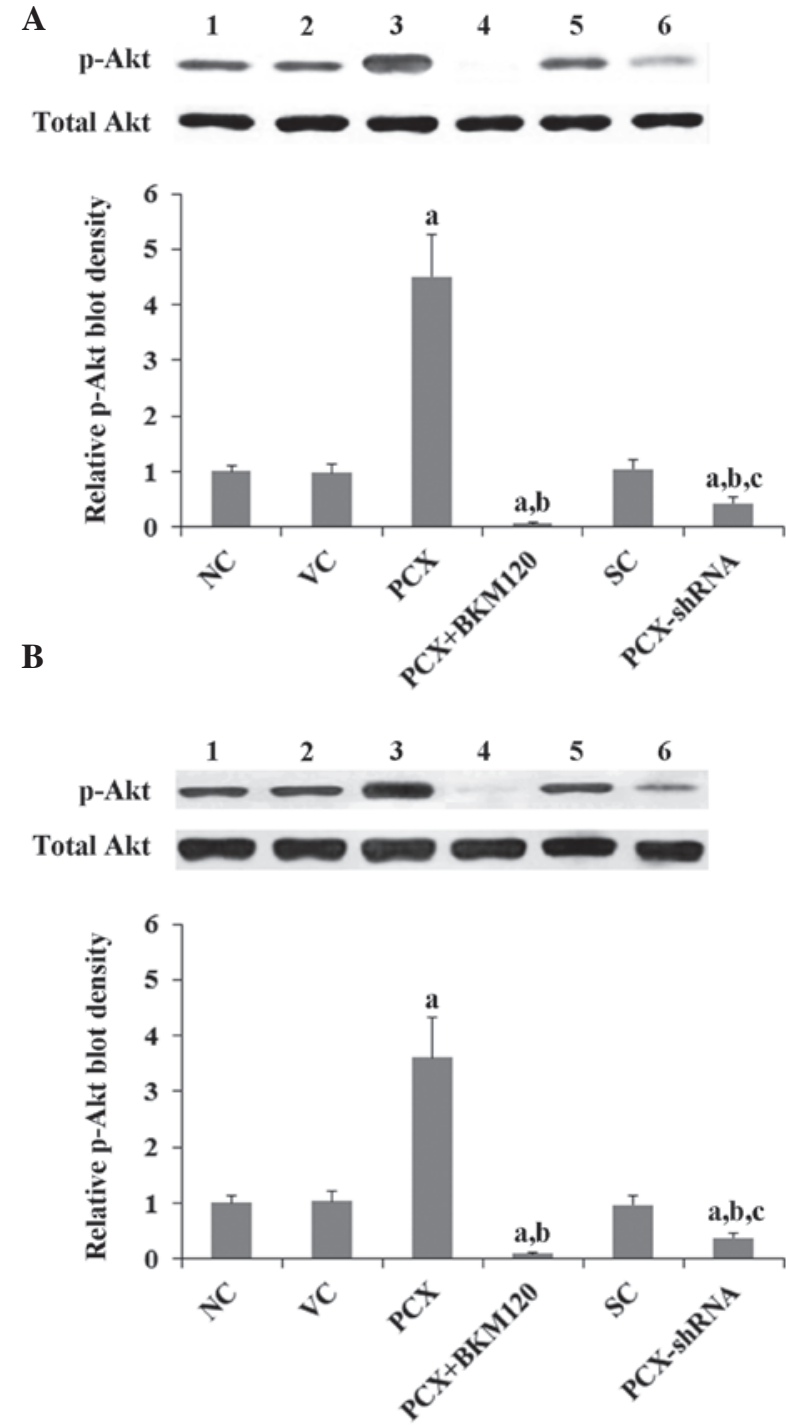

Figure 6. Effects of PCX on p-Akt expression levels in OS cells. In (A) MG-63 and (B) U2OS human OS cells, expression levels of total Akt and p-Akt at ser473 were determined by western blot analyses. Lanes: $1, \mathrm{NC} ; 2, \mathrm{VC} ; 3$, PCX; 4, PCX + BKM120; 5, SC; and 6, PCX-shRNA. Total Akt expression levels were not significantly altered by PCX in MG-63 and U2OS cells. The density of the p-Akt (ser473) blot was normalized against that of total Akt in order to obtain the relative p-Akt blot density, which was expressed as a fold changes of that of the NC (designated as 1). Three independent experiments were performed for each western blot analysis. Values are expressed as the mean + standard deviation. ${ }^{\mathrm{a}} \mathrm{P}<0.05$, vs. the controls ( $\mathrm{NC}, \mathrm{VC}$ and $\mathrm{SC})$; ${ }^{\mathrm{b}} \mathrm{P}<0.05$, vs. the $\mathrm{PCX}$ group; ${ }^{\mathrm{C}} \mathrm{P}<0.05$, vs. the $\mathrm{PCX}+\mathrm{BKM} 120$ group. Groups: NC, normal control cells; VC, cells stably transfected with empty pcDNA3.1 vector; PCX, cells stably transfected with PCX; PCX + BKM120, cells stably transfected with PCX and treated with the phosphatidylinositide 3-kinase inhibitor BKM120 $(50 \mu \mathrm{M})$ for $48 \mathrm{~h}$; SC, cells stably transduced with scramble control shRNA; and PCX-shRNA, cells stably transduced with PCX-shRNA. p-Akt, phosphorylated Akt; PCX, podocalyxin; OS, osteosarcoma; shRNA, small hairpin RNA; ser, serine.

of Akt (Fig. 6; $\mathrm{P}<0.05$ ), which is required for full activation of Akt by PI3K. Overexpression of PCX was observed to increas Akt phosphorylation by approximately 4.5 -fold in MG-63 cells and by approximately 3.6 -fold in U2OS cells, as compared with that of the control cells, the phosphorylation of which was abolished by BKM120 treatment (50 $\mu \mathrm{M})$ (Fig. 6). Conversely, knockdown of PCX expression reduced Akt phosphorylation by approximately 55\% in the two cell lines (Fig. 6).

\section{Discussion}

The use of multiagent, intensive chemotherapy has markedly improved the long-term survival rate of patients with OS (5). However, chemoresistance continues to be the principal reason for poor survival and disease recurrence in patients with OS (26). Innate or acquired resistance to cisplatin, one of the most effective drugs against OS (27), is common (28). Therefore, understanding the molecular basis for cisplatin chemoresistance in OS cells may serve as a basis for identification of novel therapeutic targets and biomarkers. A previous study suggested that PCX, a transmembrane protein that is critical for the malignant progression of various types of cancer (11-16), may also contribute to cancer chemoresistance (17). The present study was the first, to the best of our knowledge, to provide evidence supporting an important role of PCX in promoting cisplatin chemoresistance in OS cells.

The clinical significance of PCX in cancer progression has been investigated in numerous types of tumor, including uterine (29), colon (30) and breast (31) carcinomas. In uterine endometrioid adenocarcinoma, PCX expression has been shown to be correlated with tumor grade (29). Furthermore, overexpression of PCX is an independent indicator of poor outcome in breast and colorectal carcinoma $(30,31)$. Specifically in colorectal cancer, PCX expression was observed predominantly on the invasive tumor front, thus suggesting its importance in the metastatic spread of this type of cancer (30). A previous study demonstrated that PCX promotes astrocytoma cell survival against temozolomide-induced apoptotic stress (17), suggesting that PCX may also promote cancer chemoresistance. Therefore, in the present study, the role of PCX was investigated in OS cell chemoresistance, in order to provide novel insight into the functional role of PCX in cancer.

The present study used two OS cell lines with relatively large differences in their genetic background as cell models to demonstrate a generalizable role of PCX in OS cell chemoresistance to cisplatin. The $\mathrm{IC}_{50}$ of cisplatin was used as a measure of cisplatin chemoresistance, with an increased $\mathrm{IC}_{50}$ value reflecting clinical chemoresistance to cisplatin (32). In addition, cell colony formation and apoptosis assays were used to measure long-term and early cell survival against cisplatin treatment, respectively. In the two cell lines, overexpression of PCX significantly increased the $\mathrm{IC}_{50}$ of cisplatin and cell colony formation, and decreased cisplatin-induced cell apoptosis, whereas knockdown of PCX expression markedly decreased the $\mathrm{IC}_{50}$ of cisplatin and cell colony formation, and enhanced cisplatin-induced cell apoptosis. Potential OS cell chemoresistance mechanisms include dysfunctional membrane transport, resistance to apoptosis and the persistence of stem cell-like tumor cells (33). The results of the present study suggested that PCX may promote cisplatin chemoresistance in OS cells predominantly through inhibition of cisplatin-induced apoptosis and promotion of cell survival.

The promoting effect of PCX on the $\mathrm{IC}_{50}$ of cisplatin and cell survival of OS cells was markedly attenuated by treatment with a selective PI3K inhibitor, which did not affect the expression of PCX. These results suggested that PCX is able to promote cisplatin chemoresistance in OS cells, predominantly through a PI3K-dependent mechanism, which was corroborated by the results showing that knockdown of PCX expression decreased PI3K activity and PI3K-mediated Akt phosphorylation in 
OS cells. These findings are also concordant with those of previous studies, which indicated that the PI3K/Akt pathway has a critical role in cancer chemoresistance (22-25). Of note, the selective PI3K inhibitor only blocked $\sim 50 \%$ of the effect of PCX on cisplatin chemoresistance and cell survival in OS cells, suggesting the involvement of other signaling pathways, which may be identified in further studies.

Neoadjuvant therapy usually consists of methotrexate, cisplatin and doxorubicin. Post-operative chemotherapy for OS usually consists of methotrexate, cisplatin, doxorubicin, cyclophosphamide and vincristine. As one of the most common first-line chemotherapy drugs for OS, cisplatin elicits DNA repair mechanisms by crosslinking DNA, which in turn activates apoptosis when DNA repair proves impossible (34). Given the important role of cisplatin in OS chemotherapy, and the significant promoting effect of PCX on cisplatin chemoresistance in OS cells, PCX may be a potential target for overcoming chemoresistance in OS. Future studies by our group are aimed at investigating whether PCX may impact OS cell resistance to other chemotherapy agents used in neoadjuvant therapy and post-operative chemotherapy.

In conclusion, the present study was the first, to the best of our knowledge, to provide evidence suggesting that PCX promotes cisplatin chemoresistance in OS cells through a PI3K-dependent mechanism. The results of the present study provided novel insight not only into the functional role of PCX in cancer, but also into the molecular mechanisms underlying OS chemoresistance.

\section{Acknowledgements}

The present study was supported by the National Natural Science Foundation, China (grant no. 81272947).

\section{References}

1. Ottaviani G and Jaffe N: The epidemiology of osteosarcoma. Cancer Treat Res 152: 3-13, 2009.

2. Subbiah V and Kurzrock R: Phase 1 clinical trials for sarcomas: The cutting edge. Curr Opin Oncol 23: 352-360, 2011.

3. FellenberJ,BerndL,Delling G,WitteDandZahlten-Hinguranage A: Prognostic significance of drug-regulated genes in high-grade osteosarcoma. Mod Pathol 20: 1085-1094, 2007.

4. Chou AJ and Gorlick R: Chemotherapy resistance in osteosarcoma: Current challenges and future directions. Expert Rev Anticancer Ther 6: 1075-1085, 2006.

5. Wang L, Jin F, Qin A, et al: Targeting Notch1 signaling pathway positively affects the sensitivity of osteosarcoma to cisplatin by regulating the expression and/or activity of Caspase family. Mol Cancer 13: 139, 2014.

6. Abe S, Nishimoto Y, Isu K, Ishii T, Goto T; Japanese Musculoskeletal Oncology Group: Preoperative cisplatin for initial treatment of limb osteosarcoma: Its local effect and impact on prognosis. Cancer Chemother Pharmacol 50: 320-324, 2002.

7. Anninga JK, Gelderblom H, Fiocco M, et al: Chemotherapeutic adjuvant treatment for osteosarcoma: Where do we stand? Eur J Cancer 47: 2431-2445, 2011.

8. Uribe-Botero G, Russell WO, Sutow WW and Martin RG: Primary osteosarcoma of bone. Clinicopathologic investigation of 243 cases, with necropsy studies in 54. Am J Clin Pathol 67: 427-435, 1977.

9. Geller DS and Gorlick R: Osteosarcoma: A review of diagnosis, management and treatment strategies. Clin Adv Hematol Oncol 8: 705-718, 2010.

10. Nielsen JS and McNagny KM: The role of podocalyxin in health and disease. J Am Soc Nephrol 20: 1669-1676, 2009.
11. Riccioni R, Calzolari A, Biffoni M, et al: Podocalyxin is expressed in normal and leukemic monocytes. Blood Cells Mol Dis 37: 218-225, 2006

12. Kelley TW, Huntsman D, McNagny KM, Roskelley CD and Hsi ED: Podocalyxin: A marker of blasts in acute leukemia. Am J Clin Pathol 124: 134-142, 2005.

13. Yasuoka H, Tsujimoto M, Hirokawa M, et al: Podocalyxin expression in undifferentiated thyroid carcinomas. J Clin Pathol 61: 1228-1229, 2008

14. Hsu YH, Lin WL, Hou YT, et al: Podocalyxin EBP50 ezrin molecular complex enhances the metastatic potential of renal cell carcinoma through recruiting Racl guanine nucleotide exchange factor ARHGEF7. Am J Pathol 176: 3050-3061, 2010

15. Sizemore S, Cicek M, Sizemore N, Ng KP and Casey G: Podocalyxin increases the aggressive phenotype of breast and prostate cancer cells in vitro through its interaction with ezrin. Cancer Res 67: 6183-6191, 2007.

16. Somasiri A, Nielsen JS, Makretsov N, et al: Overexpression of the anti-adhesin podocalyxin is an independent predictor of breast cancer progression. Cancer Res 64: 5068-5073, 2004.

17. Wu H, Yang L, Liao D, Chen Y, Wang W and Fang J: Podocalyxin regulates astrocytoma cell invasion and survival against temozolomide. Exp Ther Med 5: 1025-1029, 2013.

18. Ding X,Zhang Z, Li S and Wang A: Combretastatin A4 phosphate induces programmed cell death in vascular endothelial cells. Oncol Res 19: 303-309, 2011.

19. Gu Y,Fan S, Xiong Y, et al: Cloning and functional characterization of TCRP1, a novel gene mediating resistance to cisplatin in an oral squamous cell carcinoma cell line. FEBS Lett 585: 881-887, 2011.

20. Cao CM, Zhang Y, Weisleder N, et al: MG53 constitutes a primary determinant of cardiac ischemic preconditioning. Circulation 121: 2565-2574, 2010.

21. Fos C, Salles A, Lang V, et al: ICOS ligation recruits the p50alpha PI3K regulatory subunit to the immunological synapse. J Immunol 181: 1969-1977, 2008.

22. Li B, Yang Y, Jiang S, Ni B, Chen $\mathrm{K}$ and Jiang L: Adenovirus-mediated overexpression of BMP-9 inhibits human osteosarcoma cell growth and migration through downregulation of the PI3K/AKT pathway. Int J Oncol 41: 1809-1819, 2012.

23. Liu ZL, Mao JH, Peng AF, et al: Inhibition of fatty acid synthase suppresses osteosarcoma cell invasion and migration via downregulation of the PI3K/Akt signaling pathway in vitro. Mol Med Rep 7: 608-612, 2013.

24. Zhao G, Cai C, Yang T, et al: MicroRNA-221 induces cell survival and cisplatin resistance through PI3K/Akt pathway in human osteosarcoma. PLoS One 8: e53906, 2013.

25. Wang TF, Wang H, Peng AF, et al: Inhibition of fatty acid synthase suppresses U-2 OS cell invasion and migration via downregulating the activity of HER2/PI3K/AKT signaling pathway in vitro. Biochem Biophys Res Commun 440: 229-234, 2013.

26. Fellenberg J, Kunz P, Sähr H and Depeweg D: Overexpression of inosine 5'-monophosphate dehydrogenase type II mediates chemoresistance to human osteosarcoma cells. PLoS One 5: e12179, 2010.

27. Janeway KA and Grier HE: Sequelae of osteosarcoma medical therapy: A review of rare acute toxicities and late effects. Lancet Oncol 11: 670-678, 2010.

28. Bruheim S, Xi Y, Ju J and Fodstad O: Gene expression profiles classify human osteosarcoma xenografts according to sensitivity to doxorubicin, cisplatin and ifosfamide. Clin Cancer Res 15: 7161-7169, 2009.

29. Yasuoka H, Tsujimoto M, Inagaki M, et al: Clinicopathological significance of podocalyxin and phosphorylated ezrin in uterine endometrioid adenocarcinoma. J Clin Pathol 65: 399-402, 2012.

30. Larsson A, Johansson ME, Wangefjord S, et al: Overexpression of podocalyxin-like protein is an independent factor of poor prognosis in colorectal cancer. Br J Cancer 105: 666-672, 2011.

31. Somasiri A, Nielsen JS, Makretsov N, et al: Overexpression of the anti-adhesin podocalyxin is an independent predictor of breast cancer progression. Cancer Res 64: 5068-5073, 2004.

32. Liu M, Wang J, Huang H, et al: miR-181a-Twist1 pathway in the chemoresistance of tongue squamous cell carcinoma. Biochem Biophys Res Commun 441: 364-370, 2013.

33. Tsai HC, Huang CY, Su HL and Tang CH: CCN2 enhances resistance to cisplatin-mediating cell apoptosis in human osteosarcoma. PLoS One 9: e90159, 2014.

34. Rosenberg B, Vancamp L, Trosko JE and Mansour VH: Platinum compounds: A new class of potent antitumour agents. Nature 222: 385-386, 1969. 